\title{
The impact of transforming healthcare delivery on cystic fibrosis outcomes: a decade of quality improvement at Cincinnati Children's Hospital
}

\author{
Christopher M Siracusa, ${ }^{1}$ Jeanne L Weiland, ${ }^{1}$ James D Acton, ${ }^{2}$ \\ Amitra K Chima, ${ }^{3}$ Barbara A Chini, ${ }^{1}$ Andrea J Hoberman, ${ }^{1}$ \\ J Denise Wetzel, ${ }^{1}$ Raouf S Amin, ${ }^{1}$ Gary L McPhail ${ }^{1}$
}

\begin{abstract}
${ }^{1}$ Division of Pulmonary Medicine, Cincinnati Children's Hospital Medical Center, Cincinnati, Ohio, USA ${ }^{2}$ Division of Pulmonary Medicine, University of Missouri Health System, Columbia, Missouri, USA ${ }^{3}$ Department of Referral Management, Cincinnati Children's Hospital Medical Center, Cincinnati, Ohio, USA
\end{abstract}

\section{Correspondence to} Dr Gary L McPhail, Division of Pulmonary Medicine, Cincinnati Children's Hospital Medical Center, MLC-2021, 3333 Burnet Avenue, Cincinnati, OH 45229-3039, USA; gary.mcphail@cchmc.org

Received 26 July 2013 Revised 3 October 2013 Accepted 4 December 2013

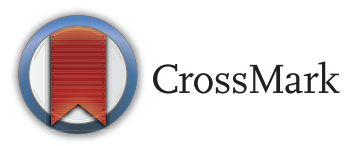

To cite: Siracusa $\mathrm{CM}$, Weiland JL, Acton JD, et al. BMJ Qual Saf 2014;23: i56-i63.

\author{
ABSTRACT \\ Background In 2001, Cincinnati Children's \\ Hospital embarked on a journey to improve \\ healthcare delivery to patients with cystic fibrosis \\ (CF). Data from the Cystic Fibrosis Foundation \\ National Patient Registry revealed our below- \\ average clinical outcomes, prompting us to \\ initiate improvement interventions.
}

Objective To improve clinical outcomes for patients with CF through a comprehensive quality-improvement approach directed at increasing patient centredness and improving healthcare delivery.

Interventions In 2001, we shared our belowaverage outcomes with patients, families and care providers. We instituted a qualityimprovement steering committee with parental and hospital leadership, and our datamanagement support was restructured to provide real-time clinical data to monitor our progress. In 2002, our weekly chart conference changed to a prospective planning session and individualised daily schedules were created for inpatients. In 2003, an influenza vaccination campaign was initiated and our infection-control practices were redesigned. In 2005, best-practice guidelines were developed for airway-clearance therapy. In 2007, evidence-based clinical algorithms were designed and implemented and key care-team members were added.

Measurements Primary outcome measures were median forced expiratory volume in $1 \mathrm{~s}$ per cent predicted (age range 6-17 years) and median body mass index percentile (age range 2-20 years).

Results From 2000 to 2010, median forced expiratory volume in $1 \mathrm{~s}$ increased from $81.7 \%$ to $100.1 \%$ predicted and median body mass index increased from the 35th to the 55th centile.

Discussion By focusing on specific outcomes, empowering families and patients, effectively using data, and standardising care processes, we transformed the culture and delivery of care for our patients with CF and learned valuable lessons potentially translatable to other chroniccare providers.

\section{INTRODUCTION}

\section{Background}

Chronic-disease outcomes improve when the healthcare delivery system provides care that is safe, patient-centred, evidencebased, efficient, timely and equitable. ${ }^{1}$ Improved outcomes are dependent on care providers who understand what is known about the pathophysiology and management of disease and have tools that help them be prepared to deliver the care that each patient needs when they need it. Chronic-disease outcomes also improve when patients and families are educated, informed and activated, thereby understanding the basic principles of disease, developing skills to minimise the impact of disease on health, and engaging in ongoing care practices that are productive and evidence-based. ${ }^{2}$

Cystic fibrosis (CF) is a life-shortening genetic disease with an incidence of approximately $1 / 3500$ births in the USA. Of the over 70000 patients with CF that have been identified worldwide, approximately 30000 of those are in the USA. For over 40 years, the Cystic Fibrosis Foundation has compiled data regarding 
patients who receive care in CF centres across the country. The median predicted age of survival for children with CF was less than 32 years in 2000 and increased to greater than 38 years in 2012. There also has been an overall improvement in lung function and nutritional outcomes at the national level, as evidenced by registry data. Since 2000 , there have been significant advances in therapy including antiinflammatory agents and inhaled antibiotics, and early recognition of CF through newborn screening has become a national standard.

\section{Setting and local problem}

Cincinnati Children's Hospital Medical Center (CCHMC) is a tertiary care pediatric hospital with a CF centre that currently cares for 250 patients with CF, 0-21 years of age. In the period between 2001 and 2010, our centre provided care to 394 individual patients.

Prior to 2000, the CF-care team at CCHMC had a strong existing commitment to deliver the best possible care and had partnered with the institution's Office of Clinical Effectiveness. However, availability of measurable outcomes was limited. Therefore, our emphasis primarily was on patient and family satisfaction. In 2000, the Cystic Fibrosis Foundation released key outcomes data from the National Patient Registry for the first time to each centre privately that allowed the centres to see how they compared with the de-identified outcomes of all other US CF centres. Although this initial data was limited and not publically transparent, for the first time it provided us a way to assess our performance and represented an important step in the evolution of quality improvement (QI) in CF care in the USA.

At the same time, the emphasis on QI at CCHMC was growing rapidly, with a charge given to directors of clinical divisions and services to develop new and innovative ways to improve outcomes. When senior CCHMC leadership applied for a Robert Wood Johnson Foundation grant, 'Pursuing Perfection: Raising the Bar for Health Care Performance', the CF-care team was invited to participate as the institution's pilot team for chronic care. This opportunity was foundational in providing us with guidance in improvement methods, as well as structure, accountability and urgency for our improvement effort, all of which accelerated the improvement in care processes and outcomes.

When the performance data for CCHMC was reviewed, it was revealed that the representative pulmonary and nutritional outcomes for our patients were below average when compared with all other US centres. Initially, the reports from the National Patient Registry were limited, with little detail to help identify specific opportunities to improve the desired outcomes. Other challenges included identifying other centres to learn from and sharing performance data with patients and families in a way that engaged them in the improvement process.

Supported by hospital leadership, we committed to CCHMC's mission to "deliver demonstrably superior patient outcomes and experiences, and discover and apply better ways to improve the health of children." Operationally, this translated into improving our access and use of data, identifying key components of $\mathrm{CF}$ care that drive the desired outcomes, and engaging all of the partners in CF care-especially patients and families-to design and implement effective changes toward improving CF outcomes.

\section{INTERVENTIONS}

\section{Ethical issues}

All clinical interventions were improvement projects, intended to close a gap in our care-delivery system. We used evidence-based care with assumed direct patient benefit and not requiring informed consent. At the time, Institutional Review Board approval was not required or even generally considered for QI work. Additionally, processes improvement was being addressed that did not involve individual patient information. However, patient privacy was a priority and was maintained throughout the development and implementation of the interventions.

\section{Planning the interventions}

Care providers and hospital leadership met in the summer of 2001 for a 'Visioning Session' during which we envisioned perfect care and decided on some key areas for improvement. These discussions were initially guided by Wagner's 'Chronic Care Model' of disease, ${ }^{2}$ to ensure that all aspects of chronic-disease management were addressed.

\section{Incorporating patients and families}

In-depth discussions occurred regarding the extent to which we wanted patients and families involved. Primarily, the team was concerned about 'airing our dirty laundry' and worried that once families heard about our less-than-average outcomes they would lose trust in their care providers and might choose to seek care at another CF centre. Another concern was that incorporating families into the care team might foster a forum for complaints that would distract from the improvement goals. The team debated having families serve as advisors by evaluating projects/processes designed by the care team versus having families at the decision table. Ultimately, it was agreed that families were to be partners with the CF-care team and serve on committees as equals, which was quite a novel idea at the time.

Several family members who we thought would be interested in improving care processes received letters explaining that there would be an interview process for selection of participating families. Those expressing interest were interviewed, and the candidates 
were reviewed. Thirteen family members ultimately joined the care team, with diversity being an important selection criterion. Family members included were a patient, mothers, fathers and grandparents, and encompassed caregivers for a variety of ages of patients. There also was a variety of disease severity represented, from new diagnosis to a parent of a child who had died of CF.

\section{Continuous review of progress}

Subsequently, a second visioning session was held with the families, where they envisioned perfect CF care. Priorities for improvement work were set, based on results from the visioning session that included families rather than the initial visioning session. All agreed that the priorities of the families needed to be the initial focus of our work. Task forces and projects were created and assigned to groups, with team members on each project that had specific interests in that project. Multiple ideas were brought to the table; some were abandoned, a few were attempted and failed, and several flourished. Each project group included at least one family member representative. Family members were trained by specialists from the Indiana Support Network and Family Voices, using Institute for Family-Centered Care principles, and trained in communication and collaboration with healthcare professionals.

Learning from other centres

In 2002, the Cystic Fibrosis Foundation shared the names of the top five best-performing CF centres, and our CF centre met with the top-ranked centres during the North American Cystic Fibrosis Conference to learn about initiatives that they had developed and implemented, thus sparking further interventions at our institution.

In the spring of 2003, key team members from our Improvement Team, including a parent, visited a topperforming centre to learn about and analyse their improvement system and initiatives, which helped our team revise planned interventions based on what did or did not work well at that centre. Almost equally

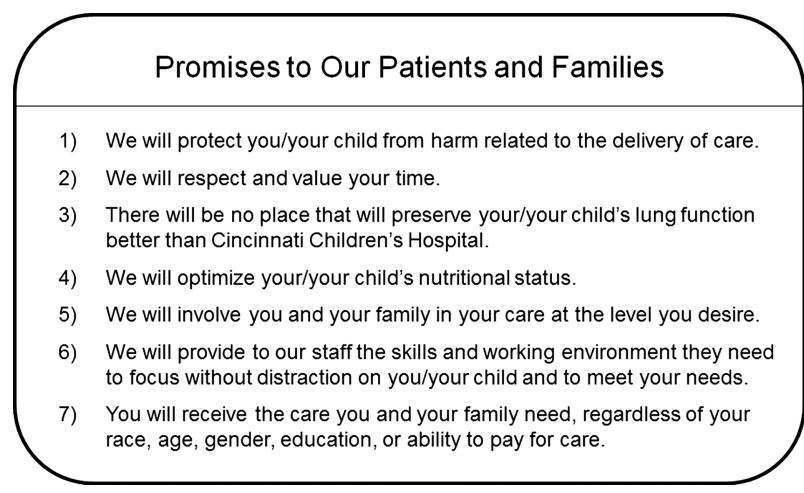

Figure 1 Promises publically made to patient and families that guided interventions. important, this site visit set into motion a cultural change that began with the motivation of our careteam members to try to make our centre one of the best in the nation.

\section{Major aims of our interventions}

After the planning phase was completed, we developed a set of promises related to providing patients with the best $\mathrm{CF}$ care possible (figure 1), and these promises became the backbone of the interventions. In general, the major aims of our endeavours included improving lung function and nutritional health outcomes for our patients by improving data collection and utilisation, standardising and redesigning care processes, and implementing cultural change. As new processes became standard of care, efforts were made to sustain existing projects and develop new ones. Patients and their families were updated periodically on our progress with a newsletter and an evening lecture series

\section{Specific interventions}

By assessing our local CF-care practices and benchmarking our practices with top-performing CF centres around the country, we used what we learned in our visioning sessions and site visits to initiate QI interventions (figure 2) designed to improve outcomes using Plan-Do-Study-Act cycles to continually evaluate the processes.

Collecting and using data more effectively

Although below-average data sparked our initiatives, it was clear that we had to use data to our advantage. Our CF centre completely revamped our local datamanagement support to provide us with real-time, trended clinical data in the form of run charts to guide interventions and monitor improvement progress.

In 2002, we changed our weekly chart conference from a retrospective review of the last week of patients, to a prospective clinic-preparation meeting. Viewing lung function and nutritional data across multiple years allowed the care-team members to develop a specific data-driven action plan for each patient coming into clinic. Although centre-level data was available annually from the Cystic Fibrosis Foundation, the team wanted real-time access to our outcomes and hired an application specialist to develop an online system called PulmonaryLink for internal data reporting and sharing. This intranet allowed the entire team to view monthly population-level data, as well as individual patient-level data (eventually stratified by risk factors in 2010) to drive QI activities and interventions. PulmonaryLink also facilitated active management of adherence to CF guidelines for care, screening and prevention by tracking which patients were due for routine office visits, pulmonary function tests and bacterial cultures. 


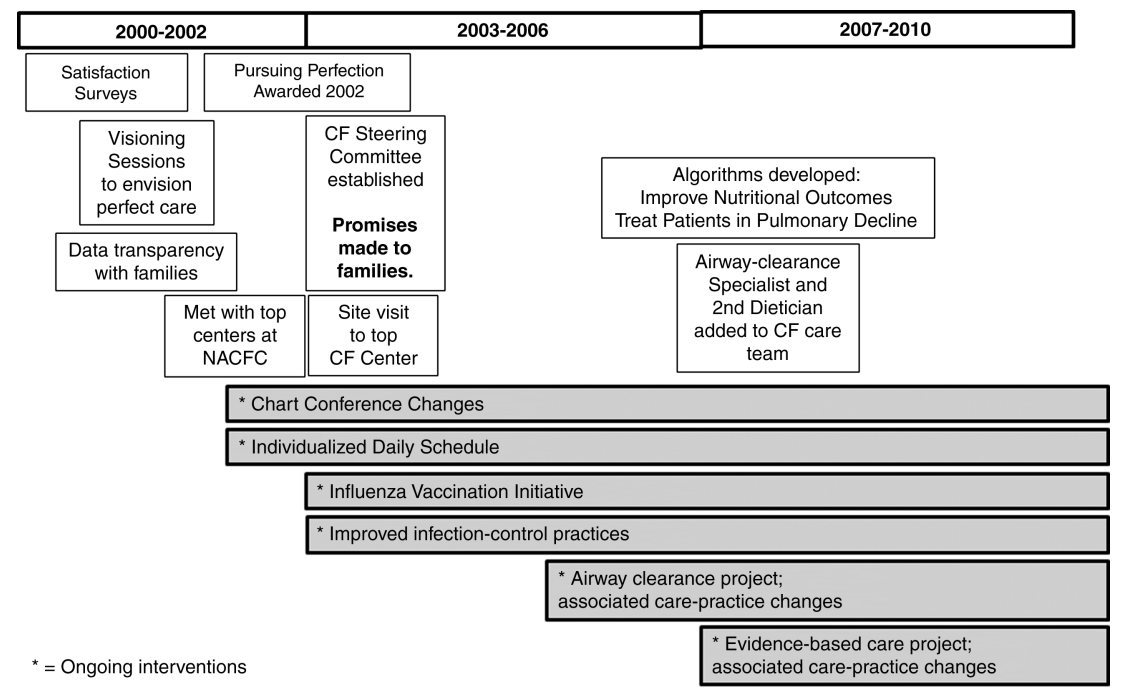

Figure 2 Timeline of initiating events and interventions.

Individualised schedules

In 2003, as a direct result of discussions at the family visioning sessions, individualised daily schedules were created for inpatient adolescents. Data on airwayclearance treatments, physical-therapy attendance and school attendance were collected. Patients who used the schedule were more likely to report that their care was often or always delivered exactly as they expected and felt that they were involved in care decisions as much as they wanted to be. When assessed, $87 \%$ of patients said using the individualised schedule made their stay a little or much better compared with past hospitalisations. ${ }^{3}$ This practice remains in place as of 2013.

Improving vaccination rates

In 2003, an Influenza Vaccination Initiative was developed and implemented. The campaign included preplanning visits, education and reminders to families, standardised order sets, and timely data tracking. There also was follow-up in place for those nonresponsive to our initial contact. Within 14 weeks, near-perfect vaccination rates were achieved at our centre (data presented at the 2004 North American Cystic Fibrosis Conference, Meeks), and these practices remain in place with continued near-perfect vaccination rates each year.

\section{Infection control}

In 2003, we redesigned our infection-control practices to decrease patient-to-patient spread of infections. Initiatives were focused on inpatient and outpatient care settings. Families, care providers and staff were educated on up-to-date infection-control practices guided by the Cystic Fibrosis Foundation, which eventually became routine. Inpatient adolescents, for a time, became 'infection control police', and they recorded how well every care provider, from physician to surgeon to nurse to housekeeper, followed the infection-control guidelines. Provider awareness of this monitoring seemed to contribute to improved adherence.

Airway clearance

In 2005, we used QI methodology to develop a series of multidisciplinary interventions to improve adherence of best-practice airway clearance therapy (ACT) in inpatient adolescents during routine clinical care. After the interventions, quality of ACT significantly improved from 21\% best-practice ACT at baseline to $73 \%$ postintervention. Quantity of ACT also significantly improved, from $41 \%$ of days with ACT four times per day at baseline to $64 \%$ after 24 months. ${ }^{4}$ We continued to collect data for an additional 12 months and concluded that the interventions were sustainable.

\section{Standardisation of care}

In 2007, evidence-based prescribing algorithms were designed to reflect consensus recommendations from the Cystic Fibrosis Foundation. Clinicians and families were educated about the guidelines, and co-leaders of this QI work met with each provider (doctor and nurse team) to review their prescribing data. Care providers often were surprised by the number of missed prescribing opportunities. Over time, we tracked adherence to prescribing practices based on the guidelines using our revamped and fully functioning database. Adherence successes and failures were highlighted at weekly team meetings to promote clinician accountability. At the start of the project, the rate of clinician adherence to prescribing guidelines was $62 \%$, which quickly improved to $87 \%$ after just 3 months. ${ }^{5}$ We continued to monitor prescribing guideline adherence for 21 months and found it to be sustained. 
Adding key team members

In 2007 , in order to facilitate an effective and equitable care model, a second registered dietician was added to the team and a role was created for an airway-clearance specialist that also was hired to join the team. In 2008, we embedded a team member whose sole responsibility was to QI and outcomes, which was an innovative concept at the time. The addition and sustainment of these members to the care team was vital to the overall success of our QI initiatives.

\section{Unsuccessful interventions}

It is important to note that there also were attempted interventions that were not successful. Early on, in an attempt to revamp our chart conference, we instituted previsit surveys mailed to families to aid in the preparation of their upcoming visit. The response to the surveys was minimal, and the project was eventually discontinued. We also attempted to initiate home spirometry testing to give us more objective data about our patients between clinic visits. However, at the time, options for home equipment were expensive for most families and unreliable.

\section{OUTCOMES}

From 2000 to 2010, lung function and nutritional health outcomes for patients with CF at CCHMC dramatically improved. Lung function outcomes, as measured by median forced expiratory volume in $1 \mathrm{~s}$ $\left(\mathrm{FEV}_{1}\right)$ per cent predicted, were determined for patients aged 6-17 years (between 95 and 118 individual patients per year) (table 1 ). $\mathrm{FEV}_{1}$ improved from $81.7 \%$ to $100.1 \%$ predicted, an absolute improvement of $18.4 \%$ predicted. Notably, our lungfunction outcome in 2010 was well above the national

Table 1 Lung function and nutritional status for patients with CF at the CCHMC CF centre, 2000-2010

\begin{tabular}{|c|c|c|c|c|}
\hline \multirow[b]{2}{*}{ Year } & \multicolumn{2}{|c|}{$\begin{array}{l}\mathrm{FEV}_{1} \text { outcomes } \\
\text { (age } 6-18 \text { years) }\end{array}$} & \multicolumn{2}{|c|}{$\begin{array}{l}\text { BMI outcomes } \\
\text { (age 2-20 years) }\end{array}$} \\
\hline & $\begin{array}{l}\text { Number of } \\
\text { patients }\end{array}$ & $\begin{array}{l}\mathrm{FEV}_{1} \text { per cent } \\
\text { predicted }\end{array}$ & $\begin{array}{l}\text { Number of } \\
\text { patients }\end{array}$ & $\begin{array}{l}\text { BMI } \\
\text { percentile }\end{array}$ \\
\hline 2000 & 115 & 81.7 & 167 & 35.41 \\
\hline 2001 & 114 & 84.6 & 164 & 44.21 \\
\hline 2002 & 106 & 85.4 & 172 & 40.24 \\
\hline 2003 & 105 & 86.5 & 164 & 45.50 \\
\hline 2004 & 95 & 89.9 & 163 & 49.63 \\
\hline 2005 & 108 & 94.3 & 169 & 47.97 \\
\hline 2006 & 115 & 95.4 & 179 & 48.63 \\
\hline 2007 & 118 & 98.2 & 181 & 54.90 \\
\hline 2008 & 115 & 97.8 & 175 & 57.59 \\
\hline 2009 & 117 & 98.8 & 182 & 57.60 \\
\hline 2010 & 114 & 100.1 & $178^{*}$ & 55.18 \\
\hline
\end{tabular}

*Denotes age range of 2-19 years for 2010. BMI, body mass index; CCHMC, Cincinnati Children's Hospital Medical Center; CF, Cystic fibrosis. average and within three percentage points of the 10 best-performing CF centres in the country (figure 3A, Source: K Petren, Cystic Fibrosis Foundation National Patient Registry).

Nutritional health outcomes, as measured by median body mass index (BMI) percentile, were recorded for patients aged 2-20 years (between 163 and 182 individual patients per year) (table 1). BMI improved from the 35 th centile in 2000 to the 55 th centile in 2010, a 1.7-fold improvement. This ranked us significantly higher than the national average of CF centres (figure 3B, Source: K Petren, Cystic Fibrosis Foundation National Patient Registry); more importantly, our outcomes were above the 50th centile benchmark designated by the Cystic Fibrosis Foundation as the minimum acceptable BMI for patients with CF.

It is notable to recognise that although health outcomes measurements for patients with $\mathrm{CF}$ across the nation were improving between 2000 and 2010, outcomes at CCHMC that had started far below the national average in 2000 had approached the top 10 centres by 2010 .

\section{DISCUSSION}

\section{Summary}

By focusing on important clinical outcomes, effectively using data, empowering families and patients, and standardising care processes, we transformed the culture and delivery of care for patients with CF at CCHMC to improve lung and nutritional outcomes and also to become a national leader in QI.

\section{Relation to other evidence}

The CF QI journey at CCHMC did not begin in a vacuum. As identified by Kaplan and colleagues in a 2010 review, $^{6}$ essential ingredients predictive of success in healthcare QI were present or developing at CCHMC in 2001, including support from hospital leadership, data infrastructure, clinician involvement in QI projects and hospital culture.

Over the past 15 years, QI literature has concluded that data and process analysis impact quality performance and hospital leadership has the strongest influence on information systems and analysis, and that these impact organisational improvement. ${ }^{7}$ In addition, local clinician leadership and collaborative involvement are a predictor of implementation success. ${ }^{8}$ A 2003 randomised controlled trial by Berner and colleagues found that having a physician leader involved in a QI programme improves adherence to the recommended interventions. ${ }^{9}$ Many studies of organisational culture and QI success have determined that creativity, risk taking, stability, attention to detail and collectivism are positively correlated with QI success.

The CF centre at CCHMC was fortunate to have many of the organisational elements essential to QI 

$2000-2010$

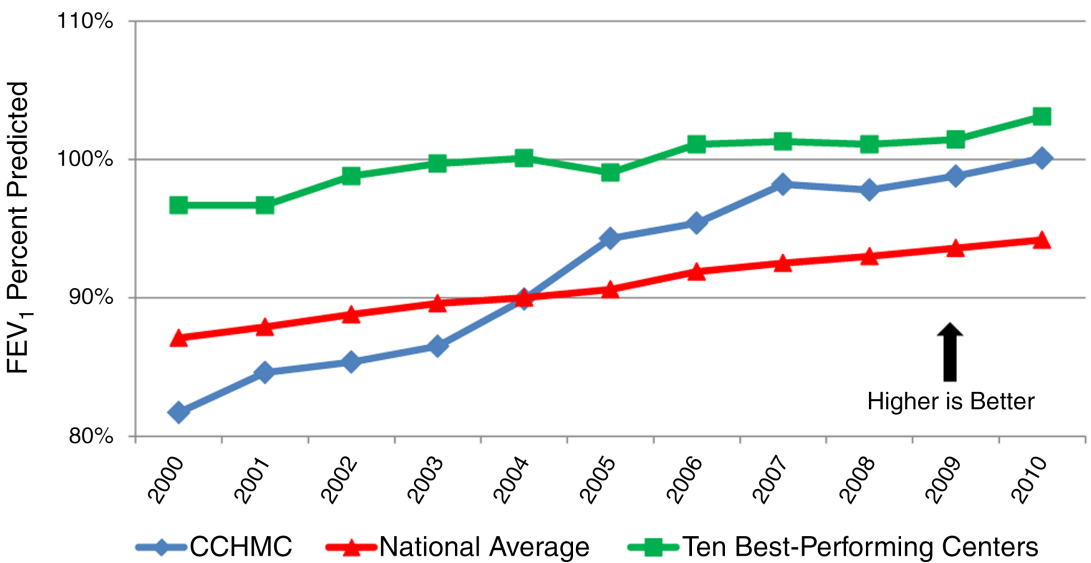

Median BMI Percentile

Cystic Fibrosis Patients 2 to 20 Years of Age 2000 - 2010

B (for 2010, age range was 2-19 years)

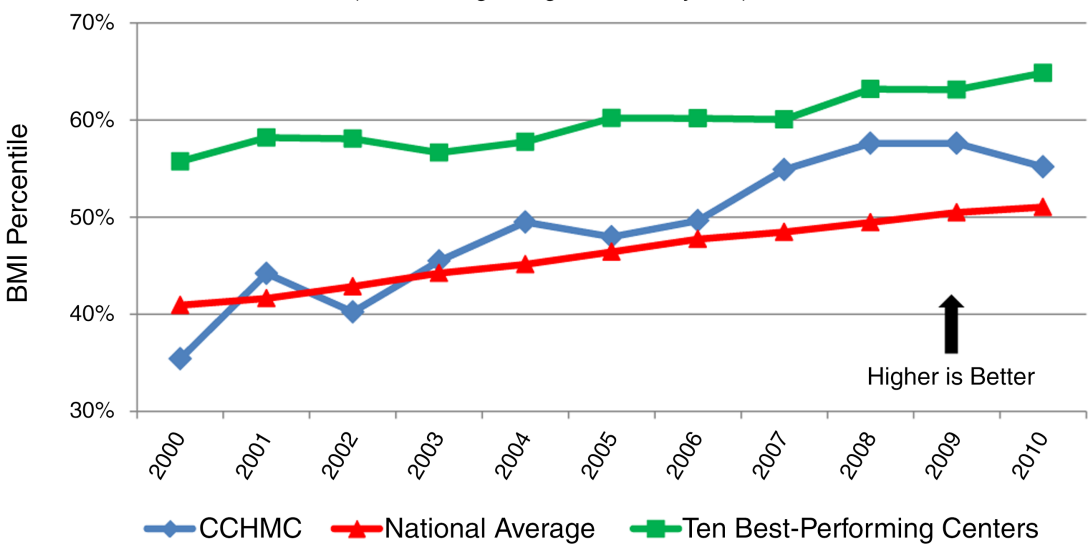

Figure 3 Cystic fibrosis (CF)-outcome improvement over a 10-year period, 2000-2010. (A) Forced expiratory volume in $1 \mathrm{~s}$ (FEV 1 ) per cent predicted and (B) Body mass index (BMI) percentile for the CF centre at CCHMC, Cincinnati Children's Hospital Medical Center (CCHMC) in comparison with the national average and the 10 best-performing CF centres nationally.

success, namely effective communication from senior leadership, direct involvement of senior leadership in QI efforts, leadership supportive of implementing formal QI instruction, and an organisational culture ready to embrace change.

\section{Conclusions}

We instituted a continuous QI programme across our $\mathrm{CF}$ centre over a decade. CCHMC is a unique clinical environment where QI is now an embedded part of the clinical culture. The transformation of our CF centre was supported by hospital leadership at multiple levels, and initially was funded by an external grant. After initial grant funding, our efforts were sustained by hospital and divisional financial support. When faced with suboptimal outcomes, our patients and families remained invested and collaborated with our staff to improve our CF centre. We dedicated resources to data management support, ensuring access to up-to-date trended data to guide our QI interventions.

National Patient Registry benchmark outcomes of $\mathrm{FEV}_{1}$ per cent predicted and median BMI percentile improved over the decade at our CF centre. This is a cross-sectional study of centre-level outcomes, not a longitudinal study of individual patient-level outcomes. The ongoing sustainability of our improvements is unknown since inevitably there will be patient and staff turnover from year to year, and therefore we will continue to track improvements over time. It is important to note that QI initiatives remain a strong focus of our CF centre and our hospital as a whole, and we are constantly striving towards our institution's doctrine to 'change the outcome' and improve the health of our patients. QI education became a main priority at CCHMC, to build improvement capability at all levels of leadership. Executive, division-level and care-team leadership engaged in 
formal, structured courses focused on developing the capability to understand improvement science and apply QI methodologies.

It is difficult to measure a change in care culture, as it is essentially an overall shift in attitude and ideals. From the board of directors to the providers, patients and families, all initiatives and care practices began to focus around one central theme: improving health outcomes by using a family-centred approach. We changed our care model and gave patients and their families a greater say in care decisions, and families became more invested in changing the outcomes, for their child, and for the overall patient population. The number of patients involved in research at our CF centre was minimal in 2000, but by 2010 almost $25 \%$ of our patients were involved in research studies.

Before 2000, like other CF centres, our healthcare team was striving to provide good care for our patients with CF, and we presumed that we were doing so. However, with the new data shared by the Cystic Fibrosis Foundation, we realised that our good intentions and hard work were not resulting in health outcomes that were up to the national average. It took courage to face these facts and not make excuses. At the beginning of the decade, we had a vision of what we wanted to achieve at our centre, though did not yet know what means were necessary to reach our goal.

During our decade of improvement, we learned several valuable lessons (figure 4). It is important to note that these experiences are not necessarily specific to CF and likely can be translated to other chronic diseases. For example, our influenza vaccination campaign was so quickly successful that it was translated to other at-risk populations with similar improvements in vaccination rates. ${ }^{10}$

It is significant to point out that CF health outcomes were, and are, continuing to improve at a national level. With the advent of new therapies and improvement of older therapies, patients are doing better than they had in past years. Another potential influence on our centre over the decade was the implementation of the newborn CF screening programme that started in 2006 in Ohio. Early recognition and treatment of CF may have contributed to our outcomes.

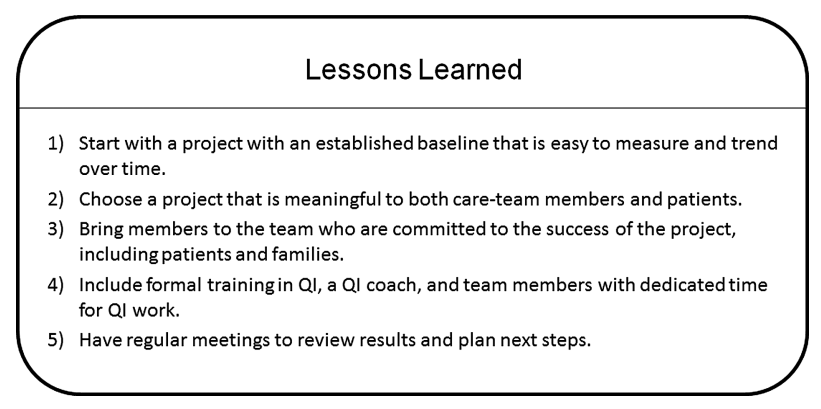

Figure 4 Lessons learned during decade of quality improvement (QI) process.
Throughout the process, there were barriers that had to be overcome for the improvement process to succeed. Initially, we encountered some team members, including those in leadership positions, who did not appreciate the value of QI work. However, sharing the data in a transparent manner helped to bring everyone on board. In addition, we sometimes overextended our resources, initiating too many QI projects at once and underscoring the importance of having team members with dedicated time for QI work. Having patients and families involved maintained motivation of the care team. Finally, it was important to celebrate achievements throughout the process to sustain morale and enthusiasm.

Our QI initiatives were effective at improving the health outcomes of patients at our CF centre and contributed to the reputation that CCHMC has as a national leader in QI. Data transparency, parent and family involvement, data-driven initiatives, and a motivated local QI team were essential features of our QI programme. QI methodology is effective for improving healthcare delivery, and changing our healthcare delivery system quickly improved our CF outcomes and transformed our culture of patient care.

Acknowledgements The authors are indebted to Jim Anderson, Thomas Boat, Lee Carter and Uma Kotagal for their long-term dedication to quality improvement, the staff of the Cystic Fibrosis Center at Cincinnati Children's Hospital Medical Center, and the patients and families who continue to participate in our improvement journey. The authors thank Maria Britto, Terri Byczkowski and Gerry Pandzik for their invaluable support and insights. The authors are indebted to Don Berwick, Tom Nolan and Lloyd Provost whose passion and expertise in improvement were critical during our journey. Thanks to Yuping Guo, Laurie Kahill and Kathy Sebastian for data/registry support. The authors also thank J P Clancy, Justin Brockbank and Greg Ogrinc for critical review of the manuscript. The authors are grateful to Terri Schindler and Robert Wilmott for their leadership in early years of the work and to Ginger Browning, Ed Conway, Monica Chapman, Geri Dinkins, Kathy Dressman, Michelle Ernst, Connie Meeks, Karen Montag, Teresa O'Hara, Adrienne Prestridge, Jackie Taylor and Jamie Wooldridge for their dedication to improve care. The authors' work would not have sustained momentum without the voices of Tracy Blackwelder, Kimberly Cook, Jim Lang, Honor Page and the other patients and families who gently reminded them that 'the clock is ticking' and that their work could not slow down because it directly impacted the lives of their children. The authors would like to thank the Cystic Fibrosis Foundation, as an integral part of their story from the beginning, and specifically Bruce Marshall, Kathy Sabadosa and David Stevens for helping promote their work and fostering the development of this supplement.

Contributors CMS: analysis and interpretation of data, primary author drafting and revising the article critically for important intellectual content, and final approval of the version published. JLW: conception and design, acquisition of data, analysis and interpretation of data, drafting and revising the article critically for important intellectual content, and final approval of the version published. JDA: conception and design, acquisition of data, analysis and interpretation of data, drafting and revising the article critically for important intellectual content, and final approval of the version 
published. AKC: conception and design, acquisition of data, analysis and interpretation of data, revising the article critically for important intellectual content, and final approval of the version published. BAC: acquisition of data, analysis and interpretation of data, drafting and revising the article critically for important intellectual content, and final approval of the version published. AJH: acquisition of data, analysis and interpretation of data, drafting and revising the article critically for important intellectual content, and final approval of the version published. JDW: analysis and interpretation of data, drafting and revising the article critically for important intellectual content, and final approval of the version published RSA: conception and design, acquisition of data, analysis and interpretation of data, revising the article critically for important intellectual content, and final approval of the version published. GLM: acquisition of data, analysis and interpretation of data, drafting and revising the article critically for important intellectual content, and final approval of the version published.

Funding Funding for these projects was provided by the Division of Pulmonary Medicine at Cincinnati Children's Hospital and through a Pursuing Perfection "Advancing the quality of health care for children and adolescents" grant by the Robert Wood Johnson Foundation (grant number 45413).

Competing interests All authors report grant funding from the Robert Wood Johnson Foundation. GLM reports personal fees from Gilead Sciences Advisory Board and from Vertex Pharmaceuticals Advisory Board outside of the submitted work. CMS reports grant funding from the Cystic Fibrosis Foundation outside of the submitted work. All other authors report no other competing interests.

Ethics approval Ethics approval was not required by our local institution. The goal of this project was an ethical imperative, to improve healthcare delivery and patient outcomes. Patients were not required to undergo additional testing. Health outcomes improved in our center by improving healthcare delivery. Patients/families consented for their clinical data to be included in the CF Registry and to be used in research.

Provenance and peer review Not commissioned; externally peer reviewed.

\section{REFERENCES}

1 Crossing the Quality Chasm: A New Health System for the 21st Century: Institute of Medicine of the National Academies, National Academy Press, Washington, D.C., 2001.

2 Wagner EH. Chronic disease management: what will it take to improve care for chronic illness? Effective Clin Pract 1998;1: 2-4.

3 Weiland J, Schoettker PJ, Byczkowski T, et al. Individualized daily schedules for hospitalized adolescents with cystic fibrosis. J Pediatr Health Care 2003;17:284-9.

4 Ernst MM, Wooldridge JL, Conway E, et al. Using quality improvement science to implement a multidisciplinary behavioral intervention targeting pediatric inpatient airway clearance. J Pediatr Psychol 2010;35:14-24.

5 McPhail GL, Weiland J, Acton JD, et al. Improving evidencebased care in cystic fibrosis through quality improvement. Arch Pediatr Adolesc Med 2010;164:957-60.

6 Kaplan HC, Brady PW, Dritz MC, et al. The influence of context on quality improvement success in health care: a systematic review of the literature. Milbank Q 2010;88: 500-59.

7 Meyer SM, Collier DA. An empirical test of the causal relationships in the Baldrige Health Care Pilot Criteria. J Operations Manag 2001;19:403-26.

8 Molfenter T, Gustafson D, Kilo C, et al. Prospective evaluation of a Bayesian model to predict organizational change. Health Care Manag Rev 2005;30:270-9.

9 Berner ES, Baker CS, Funkhouser E, et al. Do local opinion leaders augment hospital quality improvement efforts? A randomized trial to promote adherence to unstable angina guidelines. Med Care 2003;41:420-31.

10 Britto MT, Schoettker PJ, Pandzik GM, et al. Improving influenza immunisation for high-risk children and adolescents. Qual Saf Health Care 2007;16:363-8. 